\title{
ПОИСК И АНАЛИЗ CRISPR-САS СИСТЕМЫ В ШТАММЕ ESCHERICHIA COLI HS И ДЕТЕКТИРУЕМЫХ СПЕЙСЕРАМИ ЕГО CRISPR-KАССЕТЫ ФАГОВЫХ РАС МЕТОДАМИ БИОИНФОРМАТИКИ
}

\author{
Е. И. Иванова ${ }^{\natural}$, Ю. П. Джиоев ${ }^{2}$, А. Ю. Борисенко ${ }^{2}$, Н. П. Перетолчина ${ }^{2}$, Л. А. Степаненко ${ }^{2}$, А. И. Парамонов ${ }^{1}$, \\ Е. В. Григорова ${ }^{1}$, У. М. Немченко ${ }^{1}$, Т. В. Туник ${ }^{1}$, Е. А. Кунгурцева \\ 'Научный центр проблем здоровья семьи и репродукции человека, Иркутск \\ ${ }^{2}$ Институт биомедицинских технологии, Иркутский государственный медицинский университет, Иркутск
}

CRISPR-Cas система - это иммунная система прокариот, обеспечивающая защиту от чужеродных репликонов, в первую очередь вирусов и плазмид. Устойчивость к повторным инфекциям приобретается в результате включения в состав CRISPR-кассет коротких последовательностей, или спейсеров, комплементарных участкам соответствующих вирусных или плазмидных геномов. В настоящее время эшерихиозные инфекции остаются серьезной проблемой практической медицины. Вследствие их крайней устойчивости к терапии с использованием антибиотиков необходима разработка новых подходов лечения. Целью исследования был поиск структур CRISPR-Cas систем в геномной последовательности штамма Escherichia coli HS. Использовали методы программного моделирования MacSyFinder (Macromolecular System Finder, ver. 1.0.2.). Поиск точной гомологии последовательностей осуществляли посредством установленных вспомогательных пакетов makeblastdb (ver. 2.2.28), HMMER (ver. 3.0). В результате методами биоинформатики была выявлена одна CRISPR-Cas система и расшифрованы спейсерные последовательности CRISPR-кассеты у штамма Escherichia coli HS. C помощью последовательностей спейсеров CRISPR-кассеты были определены комплементарные им протоспейсерные участки нескольких типов фагов, что позволяет оценить степень их устойчивости к этим чужеродным генетическим элементам.

Ключевые слова: биоинформатика, CRISPR-Cas система, Escherichia coli HS, бактериофаги

$\bowtie$ Для корреспонденции: Елена Иннокентьевна Иванова

ул. Тимирязева, д. 16, г. Иркутск, 664003; ivanova.iem@gmail.com

Статья получена: 15.03.18 Статья принята к печати: 24.03.18

DOI: $10.24075 /$ vrgmu.2018.019

\section{THE SEARCH AND ANALYSIS OF A CRISPR-CAS SYSTEM IN ESCHERICHIA COLIHS WITH SUBSEQUENT SCANNING FOR THE CORRESPONDING PHAGE RACES BASED ON THE SPACERS OF THE DETECTED CRIPSR ARRAY USING BIOINFORMATIC METHODS}

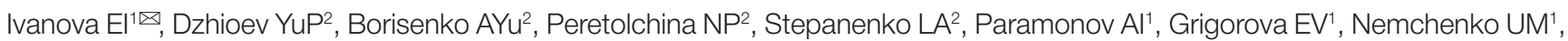
Tunik TV1 ${ }^{1}$ Kungurtseva EA ${ }^{1}$

\footnotetext{
Scientific Center for Family Health and Human Reproduction Problems, Irkutsk

${ }^{2}$ Research Institute for Biomedical Technologies of Irkutsk State Medical University, Irkutsk
}

\begin{abstract}
CRISPR-Cas is an immune system of prokaryotes that protects them against alien replicons, mainly viruses and plasmids. Short sequences (spacers) complementary to the regions of a viral or plasmid genome are inserted into a CRISPR array conferring resistance to reinfection. Infections caused by Escherichia coli still present a serious challenge for clinical medicine. The aim of this study was to scan the genome of Escherichia coli HS for CRISPR-Cas components. The search was conducted using MacSyFinder (Macromolecular System Finder, ver. 1.0.2.), a program for bioinformatic modelling. Sequence homology searches were done using makeblastdb (ver. 2.2.28) and HMMER (ver. 3.0) tools. Bioinformatics-based methods allowed us to detect one CRISPR-Cas system in the studied genome of Escherichia coli HS and read the spacer sequences of its CRIPSR array. The protospacer regions complementary to the spacer sequences of the detected CRISPR array are typical for a few types of phages. Based on these findings, one can assess the degree of bacterial resistance to alien genetic elements.
\end{abstract}

Keywords: bioinformatics, CRISPR-Cas system, Escherichia coli HS, bacteriophage

$\triangle$ Correspondence should be addressed: Elena Ivanova

Timiryazeva 16, Irkutsk, 664003; ivanova.iem@gmail.com

Received: 15.03.18 Accepted: 24.03.18

DOI: 10.24075/brsmu.2018.019

Escherichia coli — это вид, состоящий из многих биотипов. Представители некоторых из них являются комменсальными колонизаторами кишечного биотопа млекопитающих (в том числе человека), другие вызывают различные типь заболеваний. Среди патогенных возбудителей кишечных инфекций, важную роль играет энтерогеморрагическая кишечная палочка Escherichia coli O157:H7, а среди комменсальных выделяются представители биотипа E. coli HS. Вызванная штаммами E. coli O157:H7 инфекция может привести к развитию гемолитико-уремического синдрома (ГУС) с формированием почечной недостаточности. E. coli 0157:H7 - это один из серотипов, способных 
продуцировать шига-токсины [1-3]. Лечения с доказанной эффективностью не существует, и во время острой фазы заболевания рекомендуется исключительно поддерживающая терапия. Полного согласия по вопросу о необходимости применения антибиотиков для лечения инфекции, вызванной шига-токсин-продуцирующими E. coli (Stx-E. coli), до сих пор не достигнуто [4, 5]. Показано, что антибиотикотерапия на стадии гастроинтестинальной инфекции Stx-E. coli повышает (приблизительно в 17 раз) риск развития развернутой картины ГУС [6]. Был сделан вывод о том, что повреждение мембраны бактерий, индуцируемое антибиотиками, может способствовать развитию острой стадии заболевания из-за выделения токсина в больших количествах [7].

В связи с этим необходима разработка альтернативных антибиотикам подходов борьбы с бактериальными патогенами, среди которых вновь становится актуальной фаговая терапия [8-10]. Для развития этого направления необходимы фундаментальные знания генетической природы взаимоотношений между бактериями и фагами, что требует наличия у исследователя их геномных структур и новых методов их анализа. На сегодняшний день уже применяются компьютерные программные методы биоинформатики, позволяющие оперировать большими массивами геномных данных и получать новую информацию о генетической природе бактерий [11].

Кроме развития методов биоинформатики значительным событием за последние несколько лет стало открытие специфической адаптивной защитной системы у прокариот. Длительное время считалось, что бактерии беззащитнь в отношении бактериофагов, но еще в 1987 г. в геноме E. coli был обнаружен странный участок, состоящий из многочисленных повторов [12]. Лишь в 2005 г. стало известно, что разделяющие эти повторы промежуточные последовательности зачастую бывают идентичными последовательностям, найденным в геномах бактериофагов и плазмид [13, 14]. Обнаруженная структура, называемая CRISPR-Cas (Clustered Regularly Interspaced Short Palindromic Repeats - CRISPR-associated proteins, или короткие палиндромные повторы, регулярно расположенные группами с CRISPR-ассоциированными белками), является специфической адаптивной защитной системой бактерий и архей от чужеродного генетического материала (в большинстве случаев от фагов и плазмид) [15-18]. CRISPR-кассеты представляют собой набор палиндромных повторов длиной 21-47 пар нуклеотидов (п.н.), разделенных уникальными спейсерными сайтами. Спейсеры комплементарно соответствуют участкам генов фагов и плазмид, к которым бактерия демонстрирует устойчивость [13]. В непосредственной близости от CRISPR-локуса находятся саs-гены, продукты которых обеспечивают функционирование CRISPR-локусов. На сегодняшний день выделяют 3 типа CRISPR-Cas систем, различающихся по механизму действия и cas-генам, представленным в геноме [19].

Использование методов биоинформатики позволяет обнаруживать и определять CRISPR-Cas системы в геномах бактерий [20, 21]. Так, тестирование фаговых рас через структуры спейсеров с помощью биоинформационных программных методов помогает в короткие сроки определить степень устойчивости бактерий к специфичным фагам и плазмидам [22-24]. Исследования в этом направлении крайне актуальны как для решения практических задач терапии инфекционных заболеваний, так и для изучения внутривидовых и межвидовых эволюционных процессов у бактерий $[17,22]$. Однако для многих видов бактерий механизм взаимоотношений между бактериями и фагами посредством их CRISPR-Cas систем и анти-CRISPR-Cas систем соответственно остается совершенно неизученным. Поэтому на первом этапе исследований необходима разработка эффективного алгоритма биоинформационных методов поиска и анализа локусов и структур CRISPR-Cas систем в геномах бактерий и последующего скрининга фраговых рас, тестируемых через их CRISPR-кассеты. Исходя из этого, целью данной работы был поиск и исследование сайтов CRISPR-Cas систем в геноме Escherichia coli HS и последующий скрининг фаговых рас через CRISPR-кассеты бактерий посредством разработанного алгоритма поисковых методов биоинформатики.

\section{МАТЕРИАЛЫ И МЕТОДЫ}

Объектом исследования послужил штамм Escherichia coli HS, два генома которого представлены в базе данных GenBank (№ NC_009800.1, секвенирован в 2017 г. и № CP000802, секвенирован в 2014 г.). Штамм E. coli HS, представленный геномом в базе данных GenBank под № NC_009800.1, был культивирован из эталонного штамма коллекции Центра разработки вакцин (США) [25]. Для поиска CRISPR-Cas систем нами была использована последовательность генома эталонного штамма под № СР000802 [26]. Данный штамм был выделен от здорового человека, желудочно-кишечный тракт которого был колонизирован этим штаммом без каких-либо видимых клинических симптомов [25].

Для поиска сайтов CRISPR-Cas систем использовали методы программного моделирования MacSyFinder (Macromolecular System Finder, ver. 1.0.2.) [27]. Программа основана на поиске через белковый профиль геномных последовательностей, закодированных как скрытые марковские модели (СММ), которые доступны в базах данных: PFAM, TIGRFAM или PRODOM. Поиск точной гомологии последовательностей осуществляли при помощи установленных вспомогательных пакетов makeblastdb (ver. 2.2.28) и HMMER (ver. 3.0), а также выявлении структурных и функциональных характеристик обнаруженных cas-генов каждого анализируемого генома [28]. Визуализацию полученных результатов в MacSyFinder осуществляли через веб-интерфейс MacSyView. В качестве языка программирования использовали Python (ver. 2.7) [29]. Поиск расшифровки CRISPR-кассет производили при помощи онлайн-приложения «CRISPI: a CRISP RInteractive database» на Gen Ouest Biolnformatics Platform (http://genouest.org/). Геномы бактерий и архей в приложении загружены из NCBI FTP Server и обработаны в программах C и Java (ver. 1.5.0.12.) [30]. Метод обнаружения данного приложения был основан на ограничении числа максимальных совпадений. Во избежание детекции ошибочных структур фактический минимальный процент идентичности, необходимый для обнаружения, был фиксирован и составлял 60\%. Работу веб-страницы осуществляли с помощью PHP (ver. 4.3.9), Java (ver. 1.5.0.12). Идентифицировали фаги по расшифрованным спейсерным последовательностям с помощью алгоритма поиска BLASTn по базе данных GenBank-Phage [31]. Были использованы онлайн-приложения: CRISPRTarget (http:// bioanalysis.otago.ac.nz/CRISPRTarget/crispr_analysis.html), Mycobacteriophage Database (http://phagesdb.org/blast/). 


\section{РЕЗУЛЬТАТЫ ИССЛЕДОВАНИЯ}

В результате поиска в геноме штамма E. coli HS (№ CP000802) был выявлен локус одной CRISPR-Cas системы в позициях 2920652-2921839 нуклеотидных оснований (н.о.), т. е. его размер составлял 1187 н.о. По структуре эта CRISPR-Cas система была отнесена к типу CAS-Type-IE.

При помощи программы MacSyFinder были обнаружены и визуализированы участки генома E. coli HS - гены, кодирующие Cas-белки:

- обязательные (mandatory), присутствие которых в геноме указывает на наличие CRISPR-Cas системы (рис. 1);

- дополнительные (accessory), соответствующие генам, которые могут быть найдены в нескольких системах и которые трудно идентифицировать с помощью одного профиля белка, но также указывающие на наличие CRISPR-Cas системы у бактерии.

При помощи MacSyFinder удалось не только обнаружить cas-гены в CRISPR-Cas системе анализируемого генома E. coli HS, но и визуализировать полученную информацию XML через MacSyView. Пример обнаруженных cas-генов и их расположение в геноме данного штамма представлен на рис. 1.

При помощи программных пакетов HMMER (ver. 3.0) и makeblastdb (ver. 2.2.28) у обнаруженных cas-генов в каждом

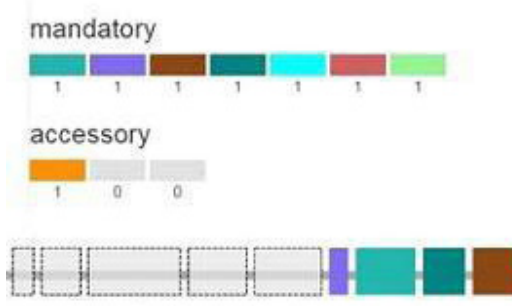

анализируемом геноме, были получены структурные и функциональные характеристики: gene (профиль), system (система, к которой принадлежит ген), hitid (идентификатор), hit seq length (длина последовательности совпадения), replicon name (имя репликона), position hit (позиция во входном файле), i-eval ("независимый Evalue"), score (оценка hit), profile coverage (процент профиля, который соответствует последовательности hit), sequence coverage (процент от последовательности hit, который соответствует профилю), begin match (начало совпадения с профилем в последовательности), end match (конец совпадения с профилем в последовательности) (рис. 2).

Расшифровку структур CRISPR-кассет осуществляли в режиме реального времени при помощи онлайнприложения «CRISPI: a CRISPR Interactive database», основанного главным образом на гомологии повторяющихся участков. В результате в CRISPRкассете исследуемого штамма было идентифицировано 11 повторяющихся (repeat) последовательностей, консенсусная схема которых представлена на рис. 3. После обнаружения повторов в приложении «CRISPI: a CRISPR Interactive database» удалось расшифровать в CRISPR-кассете 10 "спейсерных» (spacer) структур (табл. 1). При помощи Java были визуализированы CRISPR-кассета и cas-гены в геноме бактерии (рис. 4).

Pис. 1. Cas-гены (А) и их расположение в геноме (Б) E. coli HS (№ CP000802), обнаруженные при помощи MacSyFinder и визуализированные через MacSyView

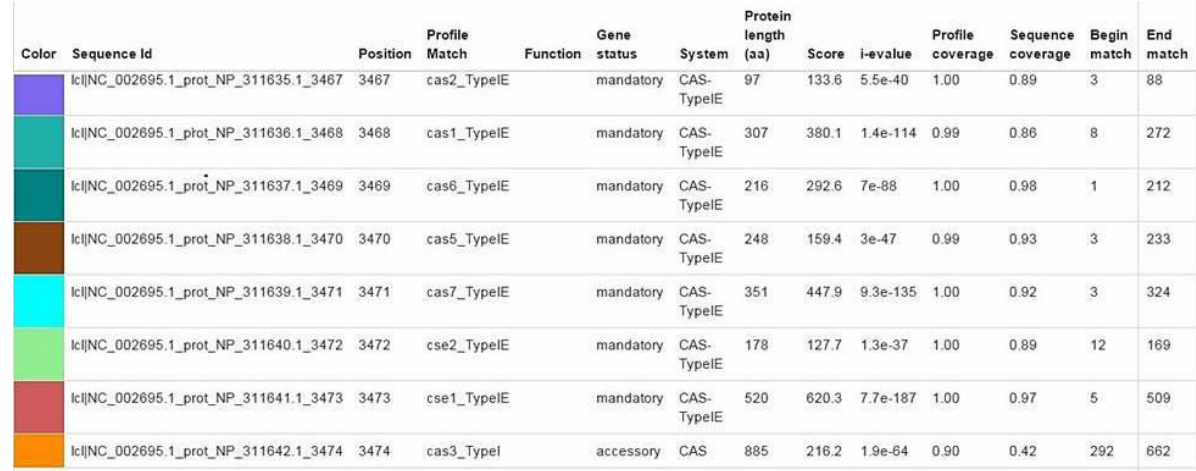

Рис. 2. Структурные и функциональные характеристики белков Cas E. coli HS (№ CP000802), обнаруженные при помощи MacSyFinder

Crispr info

\begin{tabular}{lr|}
\hline Escherichia coli O157_H7 str. Sakai chromosome & Kingdom : Bacteria \\
RefSeq :NC_002695 & 20 units \\
Consensus and repeat palindromic structure: & \\
CGGTTTATCCCCGCAGGCGCGGGGAACTC $(29 \mathrm{bp})$ & End position 2921839 \\
\hline Begin position 2920652 &
\end{tabular}

Consensus view

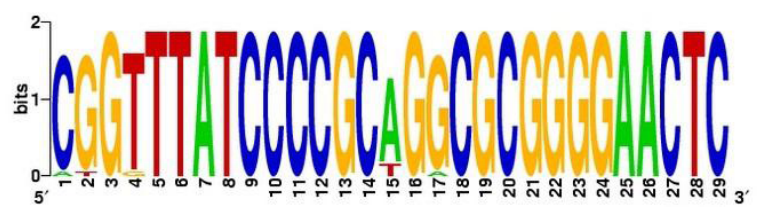

Pис. 3. Консенсусная схема чередующихся повторов в геноме штамма E. coli HS (№ CP000802), полученных при помощи приложения «CRISPI: a CRISPR Interactive database». Размер нуклеотидных символов свидетельствует о величине вариабельности нуклеотида в последовательности повтора — чем меньше его высота, тем он более изменчив 


\section{ORIGINAL RESEARCH I MICROBIOLOGY}

\section{ОБСУЖДЕНИЕ РЕЗУЛЬТАТОВ}

В прошлом году в базе данных GenBank был задокументирован геном штамма Escherichia coli HS (№ NC_009800.1) и появилась информация о наличии в его геноме трех локусов CRISPR-Cas систем. В базе данных CRISPR-Cas систем (http://crispr.i2bc.parissaclay.fr/crispr/) эти локусы представлены в нескольких вариантах. Структуры CRISPR-кассеты, полученные в нашем исследовании генома штамма E. coli HS (№ СР000802, секвенирован в 2014 г.) в основном совпадали со структурами штамма E. coli HS (вариант NC_009800_6, секвенирован в 2017 г.).

В ходе работы была проведена идентификация фрагов по расшисрованным спейсерным последовательностям, выявленным в CRISPR-кассете этого штамма (табл. 2). Из 10 спейсерных последовательностей только 4 спейсера $(1,5,7,10)$ комплементарно связались с протоспейсерными

Рис. 4. Расположение саs-генов и CRISPR-кассеты в геноме штамма E. coli HS (№ CP000802)

Таблица 1. Список последовательностей в CRISPR-кассете: спейсерные участки (spacer) разделенные повторами (repeat-unit), обнаруженные при помощи "CRISPI: a CRISPR Interactive database» в геноме штамма E. coli HS (№ CP000802)

\begin{tabular}{|c|c|c|c|c|}
\hline Спейсеры/повторы & Начало & Конец & Нуклеотидные последовательности & Размер \\
\hline unit 1 & 2920652 & 2920680 & ATGGTTATCCCCGCTGACGCGGGGAACTC & 29 \\
\hline spacer 1 & 2920681 & 2920712 & TCGTCCAGACTGAATACGTTGTCCCAAAATCT & 31 \\
\hline unit 2 & 2920713 & 2920741 & CGGTTTATCCCCGCTGGCGCGGGGAACTC & 29 \\
\hline spacer 2 & 2920742 & 2920773 & CTATTGATGAGGTGCACCATCAGAAGCGAGAT & 31 \\
\hline unit 3 & 2920774 & 2920802 & CGGTTTATCCCCGCTGGCGCGGGGAACTC & 29 \\
\hline spacer 3 & 2920803 & 2920834 & GACGTACAGATTGGCTGCGGCACCTCAAACAC & 31 \\
\hline unit 4 & 2920835 & 2920863 & CGGTTTATCCCCGCAGGCGCGGGGAACTC & 29 \\
\hline spacer 4 & 2920864 & 2920895 & TTAATTCGCGTACCTGCGCATCCATTGCCGCG & 31 \\
\hline unit 5 & 2920896 & 2920924 & CGGTTTATCCCCGCAGGCGCGGGGAACTC & 28 \\
\hline spacer 5 & 2920925 & 2920956 & CGCAATCATGTITTCATTGGGTTIACGTCCT & 31 \\
\hline unit 6 & 2920957 & 2920985 & CGGTTTATCCCCGCAGGCGCGGGGAACTC & 28 \\
\hline spacer 6 & 2920986 & 2921017 & TTTTTATGACTGAATCCACTACGCCTTCATAG & 31 \\
\hline unit 7 & 2921018 & 2921046 & CGGTTTATCCCCGCAGGCGCGGGGAACTC & 28 \\
\hline spacer 7 & 2921047 & 2921078 & TTTACGTCGTTGATGACATCGTTCAGGTGTTT & 31 \\
\hline unit 8 & 2921079 & 2921107 & CGGTTTATCCCCGCAGGCGCGGGGAACTC & 28 \\
\hline spacer 8 & 2921108 & 2921139 & GTGATITCGTACCCGGCGCGATCGCGATATG & 31 \\
\hline unit 9 & 2921140 & 2921168 & CGGTTTATCCCCGCAGGCGCGGGGAACTC & 28 \\
\hline spacer 9 & 2921169 & 2921200 & GATAACCGCTTCGCGGTCAATATCTGCCGCAC & 31 \\
\hline unit 10 & 2921201 & 2921229 & CGGTTATCCCCGCAGGCGCGGGGAACTC & 28 \\
\hline spacer 10 & 2921230 & 2921261 & GCCCATCGCCTGCGCCACACTGTTAAAAAGTT & 31 \\
\hline unit 11 & 2921262 & 2921290 & CGGTTATCCCCGCAGGCGCGGGGAACTC & 28 \\
\hline spacer 11 & 2921291 & 2921322 & TCATTCGCAATCATCCACTGACTCAGGGGCTG & 31 \\
\hline
\end{tabular}


Таблица 2. Спект фаговых рас, выявленных по комплементарным структурам спейсерных последовательностей CRISPR-кассеты штамма E. соli HS (№ CP000802)

\begin{tabular}{|c|c|c|c|}
\hline № & Спейсер & Бактериофаги & Количество замен \\
\hline 1 & спейсер 1 (2920681-2920712) & $\begin{array}{l}\text { Aeromonas phage phiAS4, (HM452125) положение: 100313-100337, } \\
\text { Cronobacter phage vB_CsaP_Ss1, (KM058087) положение: 19880-19863 }\end{array}$ & $\begin{array}{l}8 \\
10\end{array}$ \\
\hline 2 & спейсер 5 (2920925-2920956) & $\begin{array}{l}\text { Salmonella phage PVP-SE1, (GU070616) положение: 124932-124959 } \\
\text { Salmonella phage SSE-121, (JX181824) положение: 87806-87779 } \\
\text { Bacillus phage Bp8p-T, (KJ010548) положение: 144792-144820 } \\
\text { Bacillus phage Bp8p-C, (KJ010547) положение: } 144790-144818\end{array}$ & $\begin{array}{l}7 \\
7 \\
8 \\
8\end{array}$ \\
\hline 3 & спейсер 7 (2921047-2921078) & $\begin{array}{l}\text { Rhizobium phage vB_RleM_P10VF, (KM199770) положение: 93101-93076 } \\
\text { Burkholderia phage phiE255, (CP000622) положение: 17180-17211 } \\
\text { Burkholderia cenocepacia phage ВсерMu, (АY539836) положение: 30887-30918 } \\
\text { Gordonia phage GTE5, (JF923796) положение: 49708-49734 } \\
\text { Dickeya phage vB_DsoM_LIMEstone1 (НЕ600015) положение: 52018-52038 } \\
\text { Dickeya phage RC-2014, (KJ716335) положение: 27496-27516 } \\
\text { Synechococcus phage S-CAM1 (НQ634177) положение: } 189041-189018 \\
\text { Cyanophage S-SSM6b (HQ316603) положение: 161353-161374 } \\
\text { Cyanophage S-SSM4 (НQ316583) положение: 103276-103255 }\end{array}$ & $\begin{array}{l}8 \\
7 \\
7 \\
8 \\
8 \\
8 \\
9 \\
10 \\
10\end{array}$ \\
\hline 4 & спейсер 10 (2921230-2921261) & Bacteriophage RTP, (AM156909) положение: 34535-34554 & 10 \\
\hline
\end{tabular}

структурами фаговых рас, представленными в данной таблице. Выявленные фаговые расы характерны для широкого круга разнообразых бактериальных хозяев. Возможно, это следствие горизонтального переноса CRISPR-Cas систем между типами бактерий за длительный период формирования и развития у них «адаптивной иммунной системы». Необходимы дальнейшие исследования, в ходе которых будут получены новые фундаментальные знания о природе антагонистических взаимоотношений между бактериями и фагами. По детектируемым фаговым расам можно судить о степени защищенности и живучести бактерий в период эволюционного развития.

\section{ВЫВОДЫ}

Проведенные поиск и анализ расшифрованной структуры CRISPR-кассеты в геноме штамма E. coli HS (№ CP000802, секвенирован в 2014 г.) позволяют считать используемые биоинформационные программные методы поиска структур
CRISPR-Cas систем в расшифрованных геномах бактерий весьма эффективными, а результаты высоко информативными. Наличие Cas-белков, относящихся к категории mandatory, свидетельствует о высокой антисаговой активности CRISPR-Cas системы изученного штамма. Количество выявленных спейсеров свидетельствует о длительности его эволюционной истории. Сравнительный анализ структур спейсеров в двух CRISPR-кассетах, выявленных в геноме штамма E. coli HS (№ CP000802, секвенированного в 2014 г. и № NC_009800.1, секвенированного в 2017 г.), показывает, что количество спейсеров в CRISPRкассете генома штамма № NC_009800.1 увеличилось до 19. При этом количество спейсеров в геноме штамма № СР000802 равно всего 10. Можно предположить, что такое увеличение количества спейсеров стало возможным за счет их накопления в процессах частого пассирования этого штамма либо вследствие большого числа случаев его контаминации фагами. В любом случае оно может свидетельствовать о высокой активности CRISPR-Cas системы штамма E. coli HS.

\section{Литература}

1. Trachtman H, Austin C, Lewinski M, Stahl R. A. Renal and neurological involvement in typical Shiga toxin-associated HUS. Nature Reviews Nephrology. 2012; (8): 658-69.

2. Croxen MA, Law RJ, Scholz R, Keeney KM, Wlodarska M, Finlay BB. Recent advances in understanding enteric pathogenic Escherichia coli. Clinical Microbiology Reviews. 2013; (26): 82280.

3. Tarr G, Shringi S, Phipps Al, Besser TE, Mayer J, Oltean HN, et al. Geogenomic Segregation and Temporal Trends of Human Pathogenic Escherichia coli O157:H7, Washington, USA, 20052014. Emerg Infect Dis. 2018; 24 (1): 32-9.

4. Ivanova El, Rychkova LV, Nemchenko UM, Bukharova EV, Savelkaeva MV, Dzhioeva YuP. The Structure of the Intestinal Microbiota of the Intestine and the Frequency of Detection of Pathogenicity Genes (stx1, stx2, bfp) in Escherichia coli with Normal Enzymatic Activity Isolated from Children during the First Year of Life. Molecular Genetics, Microbiology and Virology. 2017; 32 (1): 42-8.

5. Holmes A, Dallman TJ, Shabaan S, Hanson M, Allison L. Validation of Whole-Genome Sequencing for Identification and Characterization of Shiga Toxin-Producing Escherichia coli To Produce Standardized Data To Enable Data Sharing. J Clin Microbiol. 2018; 56 (3): e01388-17.

6. Wong CS, Mooney JC, Brandt JR, Staples AO, Jelacic S, Boster
$\mathrm{DR}$, et al. Risk factors for the hemolytic uremic syndrome in children infected with Escherichia coli O157:H7: a multivariable analysis. Clin Infect Dis. 2012; 55 (1): 33-41.

7. Freedman SB, Xie J, Neufeld MS, Hamilton WL, Hartling L, Tarr PI Shiga toxin-producing Escherichia coli infection, antibiotics, and risk of developing hemolytic uremic syndrome: A meta-analysis. Clinical Infectious Diseases. 2016; (62): 1251-58.

8. Pirnay JP, Blasdel BG, Bretaudeau L, Buckling A, Chanishvili N, Clark JR, et.al. Quality and safety requirements for sustainable phage therapy products. Pharm Res. 2015; 32 (7): 2173-79.

9. Qadir Ml. Review: phage therapy: a modern tool to control bacterial infections. Pak J Pharm Sci. 2015; 28 (1): 265-70.

10. Назаров П. А. Альтернативы антибиотикам: литические ферменты бактериофагов и фаговая терапия. Вестник РГМУ 2018; (1).

11. Rusconi B, Sanjar F, Koenig SS, Mammel MK, Tarr PI, Eppinger M. Whole Genome Sequencing for Genomics-Guided Investigations of Escherichia coli O157:H7 Outbreaks. Front Microbiol. 2016; 7: 985.

12. Nakata A, Amemura M, Makino KJ. Unusual nucleotide arrangement with repeated sequences in the Escherichia coli K-12 chromosome. Bacteriol. 1989; 171 (6): 3553-56.

13. Bolotin A, Quinquis B, Sorokin A, Ehrlich SD. Clustered regularly interspaced short palindrome repeats (CRISPRs) have spacers of 
extrachromosomal origin. Microbiology 2005; 151: 2551-61.

14. Brouns SJ, Jore MM, Lundgren M, Westra ER, Slijkhuis RJ, Snijders AP, et al. CRISPR RNAs guide antiviral defense in prokaryotes. Science. 2008; (321): 960-4.

15. Джагаров Д. Э. Умные ножницы для ДНК. Химия и жизнь. 2014; (7): 6-9.

16. Westra ER, Buckling A, Fineran PC. CRISPR-Cas systems: beyond adaptive immunity. Nat Rev Microbiol. 2014; 12 (5): $317-$ 26.

17. Makarova KS, Koonin EV. Annotation and Classification of CRISPR-Cas Systems. Methods Mol Biol. 2015; (1311): 47-75.

18. Modell JW, Jiang W, Marraffini LA. CRISPR-Cas systems exploit viral DNA injection to establish and maintain adaptive immunity. Nature. 2017; 544 (7648): 101-4.

19. Gasiunas G, Sinkunas T, Siksnys V. Molecular mechanisms of CRISPR-mediated microbial immunity. Cellular and Molecular Life Sciences. 2014; 71 (3): 449-65.

20. Peretolchina NP, Dzhioev YP, Boricenko AY, Paramonov Al, Voskresenskaya EA, Stepanenko LA, et al. Bioinformatic search and screening of phages and plasmids via spaser sites of Yersinia pseudotuberculosis YPIII CRISPR/Cas System. Materials 15th Medical Biodefence Conference, 26-29 April 2016; Minich: 4344.

21. Zlobin VI, Dzhioev YuP, Peretolchina NP, Borisenko AY, Stepanenko LA, Yingchen Wang, et.al. Prospects to Enhance Phage Therapy by Looking At CRISP Fingerprints in Bacterial Populations. Current Trends in Biomedical Engineering \& Biosciences. 2018; 10 (5): $1-3$

22. Abedon S, Kuhl S, Blasdel B. Phage treatment of human infections. Bacteriophage. 2011; (1): 66-85.

23. Степаненко Л. А., Джиоев Ю. П., Борисенко А. Ю., Колбасеева О. В., Злобин В. И., Малов И. В. Поиск фагов и плазмид через спейсерные сайты CRISPR/CAS-системы
Neisseria meningitides fdaargos_214. Журнал инфектологии. 2018; 10 (S1): 30-31.

24. Борисенко А. Ю., Джиоев Ю. П., Перетолчина Н. П., Степаненко Л. А., Кузьминова В. М., Колбасеева О. В. и др. Биоинформационный поиск и скрининг бактериофагов через спейсеры CRISPR/CAS-системы штамма Staphylococcus aureus Mu3. Актуальные проблемы науки прибайкалья. 2017; (2): 45-9.

25. Levine M. M., Rennels M. B. E. coli colonization factor antigen in diarrhoea. Lancet. 1978; 2 (8088): 534.

26. Rasko DA, Rosovitz MJ, Myers GSA, Mongodin EF, Fricke WF, Gajer P, et al. The Pangenome Structure of Escherichia coli: Comparative Genomic Analysis of E. coli Commensal and Pathogenic Isolates. J Bacteriol. 2008; 190 (20): 6881-93.

27. Abby SS, Néron B, Ménager H, Touchon M, Rocha Eduardo PC. MacSyFinder: A Program to Mine Genomes for Molecular Systems with an Application to CRISPR-Cas Systems. PLoS One. 2014; 9 (10): e110726.

28. Gaj T, Gersbach CA, Barbas CF. ZFN, TALEN and CRISPR/Casbased methods for genome engineering. Trends Biotechnol. 2013; 31: 397-405.

29. Qi LS, Larson MH, Gillbert LA, Doudna JA, Weissman JS, Arkin AP, et al. Repurposing CRISPR as an RNA guided platform for sequence-specific control of gene expression. Cell. 2013; 152 (5): 1173-83.

30. Grissa I, Vergnaud G, Pourcel C. CRISPRFinder: a web tool to identify clustered regularly interspaced short palindromic repeats. Nucleic Acids Res. 2007; (35) (Web Server issue): W52-7.

31. Johnson M, Zaretskaya I, Raytselis $Y$, Merezhuk $Y$, McGinnis S, Madden TL. NCBI BLAST: a better web interface. NCBI BLAST: a better web Interface. Nucleic Acids Res. 2008; (1); 36 (Web Server issue): W5-9.

\section{References}

1. Trachtman $\mathrm{H}$, Austin C, Lewinski M, Stahl R. A. Renal and neurological involvement in typical Shiga toxin-associated HUS Nature Reviews Nephrology. 2012; (8): 658-69.

2. Croxen MA, Law RJ, Scholz R, Keeney KM, Wlodarska M, Finlay BB. Recent advances in understanding enteric pathogenic Escherichia coli. Clinical Microbiology Reviews. 2013; (26): 82280.

3. Tarr G, Shringi S, Phipps Al, Besser TE, Mayer J, Oltean HN, et. al. Geogenomic Segregation and Temporal Trends of Human Pathogenic Escherichia coli O157:H7, Washington, USA, 20052014. Emerg Infect Dis. 2018; 24 (1): 32-9.

4. Ivanova El, Rychkova LV, Nemchenko UM, Bukharova EV Savelkaeva MV, Dzhioeva YuP. The Structure of the Intestina Microbiota of the Intestine and the Frequency of Detection of Pathogenicity Genes (stx1, stx2, bfp) in Escherichia coli with Normal Enzymatic Activity Isolated from Children during the First Year of Life. Molecular Genetics, Microbiology and Virology. 2017; 32 (1): 42-8

5. Holmes A, Dallman TJ, Shabaan S, Hanson M, Allison L. Validation of Whole-Genome Sequencing for Identification and Characterization of Shiga Toxin-Producing Escherichia coli To Produce Standardized Data To Enable Data Sharing. J Clin Microbiol. 2018; 56 (3): e01388-17.

6. Wong CS, Mooney JC, Brandt JR, Staples AO, Jelacic S, Boster DR, et al. Risk factors for the hemolytic uremic syndrome in children infected with Escherichia coli O157:H7: a multivariable analysis. Clin Infect Dis. 2012; 55 (1): 33-41.

7. Freedman SB, Xie J, Neufeld MS, Hamilton WL, Hartling L, Tarr PI. Shiga toxin-producing Escherichia coli infection, antibiotics, and risk of developing hemolytic uremic syndrome: A meta-analysis. Clinical Infectious Diseases. 2016; (62): 1251-58.

8. Pirnay JP, Blasdel BG, Bretaudeau L, Buckling A, Chanishvili N, Clark JR, et.al. Quality and safety requirements for sustainable phage therapy products. Pharm Res. 2015; 32 (7): 2173-79.

9. Qadir Ml. Review: phage therapy: a modern tool to control bacterial infections. Pak J Pharm Sci. 2015; 28 (1): 265-70.

10. Nazarov PA. Al'ternativy antibiotikam: liticheskie fermenty bakteriofagov i fagovaja terapija. Vestnik RGMU. 2018; (1).

11. Rusconi B, Sanjar F, Koenig SS, Mammel MK, Tarr PI, Eppinger M. Whole Genome Sequencing for Genomics-Guided Investigations of Escherichia coli 0157:H7 Outbreaks. Front Microbiol. 2016; 7: 985

12. Nakata A, Amemura $M$, Makino KJ. Unusual nucleotide arrangement with repeated sequences in the Escherichia coli K-12 chromosome. Bacteriol. 1989; 171 (6): 3553-56.

13. Bolotin A, Quinquis B, Sorokin A, Ehrlich SD. Clustered regularly interspaced short palindrome repeats (CRISPRs) have spacers of extrachromosomal origin. Microbiology 2005; 151: 2551-61.

14. Brouns SJ, Jore MM, Lundgren M, Westra ER, Slijkhuis RJ, Snijders AP, et al. CRISPR RNAs guide antiviral defense in prokaryotes. Science. 2008; (321): 960-4.

15. Dzhagarov DJe. Umnye nozhnicy dlja DNK. Himija i zhizn'. 2014; 7: 6-9.

16. Westra ER, Buckling A, Fineran PC. CRISPR-Cas systems: beyond adaptive immunity. Nat Rev Microbiol. 2014; 12 (5): $317-$ 26.

17. Makarova KS, Koonin EV. Annotation and Classification of CRISPR-Cas Systems. Methods Mol Biol. 2015; (1311): 47-75.

18. Modell JW, Jiang W, Marraffini LA. CRISPR-Cas systems exploit viral DNA injection to establish and maintain adaptive immunity. Nature. 2017; 544 (7648): 101-4.

19. Gasiunas G, Sinkunas T, Siksnys V. Molecular mechanisms of CRISPR-mediated microbial immunity. Cellular and Molecular Life Sciences. 2014; 71 (3): 449-65.

20. Peretolchina NP, Dzhioev YP, Boricenko AY, Paramonov Al, Voskresenskaya EA, Stepanenko LA, et al. Bioinformatic search and screening of phages and plasmids via spaser sites of Yersinia pseudotuberculosis YPIII CRISPR/Cas System. Materials 15th Medical Biodefence Conference, 26-29 April 2016; Minich: 4344.

21. Zlobin VI, Dzhioev YuP, Peretolchina NP, Borisenko AY, Stepanenko LA, Yingchen Wang, et.al. Prospects to Enhance 
Phage Therapy by Looking At CRISP Fingerprints in Bacterial Populations. Current Trends in Biomedical Engineering \& Biosciences. 2018; 10 (5): 1-3.

22. Abedon S, Kuhl S, Blasdel B. Phage treatment of human infections. Bacteriophage. 2011; (1): 66-85.

23. Stepanenko LA, Dzhioev JuP, Borisenko AJu, Kolbaseeva OV, Zlobin VI, Malov IV. Poisk fagov i plazmid cherez spejsernye sajty CRISPR/CAS-sistemy Neisseria meningitides fdaargos_214. Zhurnal infektologii. 2018; 10 (S1): 30-31.

24. Borisenko AJu, Dzhioev JuP, Peretolchina NP, Stepanenko LA, Kuz'minova VM, Kolbaseeva OV i dr. Bioinformacionnyj poisk i skrining bakteriofagov cherez spejsery CRISPR/CAS-sistemy shtamma Staphylococcus aureus Mu3. Aktual'nye problemy nauki pribajkal'ja. 2017; 2: 45-9.

25. Levine M. M., Rennels M. B. E. coli colonization factor antigen in diarrhoea. Lancet. 1978; 2 (8088): 534.

26. Rasko DA, Rosovitz MJ, Myers GSA, Mongodin EF, Fricke WF, Gajer P, et al. The Pangenome Structure of Escherichia coli: Comparative Genomic Analysis of E. coli Commensal and
Pathogenic Isolates. J Bacteriol. 2008; 190 (20): 6881-93.

27. Abby SS, Néron B, Ménager $H$, Touchon M, Rocha Eduardo PC. MacSyFinder: A Program to Mine Genomes for Molecular Systems with an Application to CRISPR-Cas Systems. PLoS One. 2014; 9 (10): e110726.

28. Gaj T, Gersbach CA, Barbas CF. ZFN, TALEN and CRISPR/Casbased methods for genome engineering. Trends Biotechnol. 2013; 31: 397-405

29. Qi LS, Larson MH, Gillbert LA, Doudna JA, Weissman JS, Arkin AP, et al. Repurposing CRISPR as an RNA guided platform for sequence-specific control of gene expression. Cell. 2013; 152 (5): 1173-83.

30. Grissa I, Vergnaud G, Pourcel C. CRISPRFinder: a web tool to identify clustered regularly interspaced short palindromic repeats. Nucleic Acids Res. 2007; (35) (Web Server issue): W52-7.

31. Johnson M, Zaretskaya I, Raytselis Y, Merezhuk Y, McGinnis S, Madden TL. NCBI BLAST: a better web interface. NCBI BLAST: a better web Interface. Nucleic Acids Res. 2008; (1); 36 (Web Server issue): W5-9. 